\title{
EL CALENDARIO DE JESÚS DE NAZARET
}

\author{
José Marcel CÁMPORA-VILLAGRÁN ${ }^{1}$
}

\begin{tabular}{lll}
\hline Recibido & $:$ & 30.04 .2020 \\
Aceptado & $:$ & 29.06 .2020 \\
Publicado & $:$ & 06.07 .2020 \\
\hline
\end{tabular}

RESUMEN: El propósito de este documento es plantear el uso de otras maneras para determinar fechas antiguas tales como la pascua de crucifixión, y proponer nuevas bases del calendario judío. Para ello, se propone emplear otra forma para determinar Lunas nuevas y la intercalación de un décimo tercer mes, con el fin de probar las declaraciones escritas en textos antiguos de testimonios acerca de Jesús de Nazaret. Los resultados llevan a confirmar la fecha de crucifixión y a plantear que Jesús se basaba en otro calendario diferente a los de su época. Ese calendario se redescubre debido a una estrecha relación entre el movimiento lunar y sus días de descansos durante su vida. Él observaba las Lunas nuevas según las fases que proclamaba el tribunal del antiguo ritual del Segundo Templo, y guardaba el séptimo día en una forma lunar.

Palabras claves: Calendario judío, crucifixión, Jesús histórico, lunas nuevas, datación.

\section{Jesus 'calendar of Nazareth}

ABSTRACT: The purpose of this document is to propound the use of other ways to determine ancient dates such as the Passover of crucifixion, and proposes new bases of the Jewish calendar. For this, it is proposed to use another way to determine New moons and the intercalation of a thirteen month, in order to test the written statements in ancient texts about testimonies of Jesus of Nazareth. The results lead to confirm the date of crucifixion, and to suggest that Jesus was based on a different calendar from those of his time. That calendar is rediscovered due to a close relationship between the lunar movement and its rest days during his life. He observed New moons according to lunar phases proclaimed by the Court of the ancient ritual of the Second Temple, and he kept the seventh day in a lunar form.

Keywords: Jewish calendar, crucifixion, historical Jesus, new moons, data.

\footnotetext{
${ }^{1}$ Universidad Aconcagua. Email: jose.campora@uaconcagua.cl
} 


\section{Journal of the Academy $|83|$}

\section{INTRODUCCIÓN}

Año tras año millones de personas recuerdan la pascua de crucifixión. Pero si Jesús de Nazaret fue un personaje histórico, hay varias preguntas pendientes: ¿cuándo murió?, ¿es posible saber algunas de sus fechas?, entre los variados calendarios ¿cuál guió su vida? En este artículo se propone una metodología que permita encontrar algunas de esas respuestas fuera de las creencias.

Además, este trabajo persigue divulgar otras bases distintas sobre las cuales sustentar el antiguo calendario del Segundo Templo de Jerusalén. Para esto, en los próximos párrafos, será necesario previamente referir un marco teórico, con algunas precisiones, y breves explicaciones.

Planteado el problema central, el paso siguiente será pasar a nuevas interrogantes específicas, antes de ofrecer respuesta general. La observación, hipótesis, la recolección de datos y la experimentación, darán paso a resultados necesarios para proponer cómo determinar las antiguas fechas que se seguían bajo el antiguo calendario hebreo vigente hasta al año 70.

Sobre la base de esas reformulaciones, se identificarán maneras de aportar evidencias en favor de la fecha de pascua de crucifixión.

Finalmente, nuevas observaciones, hipótesis, experimentos y evaluación, darán como resultados proponer una teoría del calendario de Jesús histórico, como modelo explicativo, de los datos internos de los evangelios.

\section{DESARROLLO}

\section{Marco teórico y planteamiento del problema central.}

Para la organización de la vida en las modernas sociedades, el tiempo se contabiliza en general en occidente desde la bula papal Inter gravissimas a partir del año 1.582 bajo el calendario gregoriano. Este es fruto de complejos procesos filosóficos, históricos y de cómputos previos, basados en calendarios lunares y solares desde los antiguos egipcios, babilónicos, griegos, hebreos, entre otros.

Para el historiador José Lull (2006), en Egipto, en -2782 estaba en aplicación un calendario civil de 365 días por año, dividido en doce meses de treinta días cada uno, más cinco días adicionales conocidos como epagónemos. También el calendario lunar se habría estado en Egipto durante el V milenio, cuyo computo de inicio del año habría de iniciarse alrededor del mes de julio (Lull, 86, 88).

Por otra parte, Lull explica que desde la antigüedad:

... los egipcios habían llegado a la deducción de que 25 años civiles corresponden a 309 meses lunares. 25 años civiles ( 365 x 25) son 9125 días, y 309 meses lunares sinódicos medios de 29,53059 días son 9124,95231 días; es decir, únicamente una hora menos que los 25 años civiles. Este pequeño desfase sólo produciría un error de un día cada 500 años...

... De esos 25 años, 16 cubren periodos de doce meses lunares mientras que los nueve restantes contienen trece meses lunares cada uno. Estos años largos corresponden en la tabla a los años 1, 3, 6, 9, 12, 17, 20 y 23 (Lull, 92, 93).

Así quedaría plasmado según el esquema reconstructivo en el calendario del papiro démotico de Carlsberg (Lull, 93; Ben-Dov 2012, 179).

En otro orden y con algunas diferencias, el calendario lunisolar era el principal sistema en la mayoría de las culturas del mediterráneo y creciente fértil, como los babilónicos o asirios. Ellos usaban el 


\section{Journal of the Academy | $84 \mid$}

momento que fuera vista por primera vez la creciente lunar tras el ocaso del sol después de la conjunción para el cómputo de sus meses. Tal como en el caso de los hebreos (Lull, 84).

Por otro lado, los griegos basados en el ciclo metónico, dedujeron -al igual que los babilónicos- que 19 años equivalían a 235 meses lunares (Lull, 93) organizando a $\operatorname{los} 1^{\circ}, 3^{\circ}, 4^{\circ}, 6^{\circ}, 7^{\circ}, 9^{\circ}, 11^{\circ}, 12^{\circ}, 14^{\circ}$, $15^{\circ}, 17^{\circ}$ y $19^{\circ}$ como años de 12 meses lunares, y a los $2^{\circ}, 5^{\circ}, 8^{\circ}, 10^{\circ}, 13^{\circ}, 16^{\circ}$ y $18^{\circ}$, de 13 meses.

La historia recuerda que Julio Cesar decidió cambiar el antiguo calendario lunar en el -46. Para ello se importó el sistema civil egipcio en versión mejorada, con días, meses y años fijos, lo que conllevó a modificar el conteo del uso de días y horas en todo el imperio, incluida también la provincia romana de Judea.

Por otra parte, dentro de la Palestina del primer siglo estaba vigente el calendario pompeyano, principalmente en las ciudades en Decápolis, como el calendario seléucida en otras ciudades helenistas. Tras los hallazgos de los rollos de Qumrán en 1947, se descubrió que durante los tiempos bíblicos había diferentes calendarios. Este hecho dejó en dudas cuál es el calendario hebreo que rigió en el antiguo Israel. Quedó claro que para la comunidad sectaria de Qumrán, era uno según las descripciones del libro de los jubileos. Dicho calendario de 364 días cada año, de 12 meses fijos de 30 días, más 4 días (Roitman 2016, 210) estacionales ("tekufah") que se podrían vincular a los dos días de equinoccios y a los dos días de solsticios, respectivamente.

Luego el calendario del libro de los jubileos habría sido aplicado además en otras distintas partes. Y esto ha llevado a proponer que el propio Jesús habría guardado tal calendario solar, celebrando por eso mismo una pascua antes que la del día oficial declarada por el Templo (Jaubert 1965, 64).

Importante es recordar que el Jesús histórico sería parte de un movimiento especial de renovación en el judaísmo de su época. Y dentro de su estudio, varios han opinado que Jesús, y junto a él, Mateo, Marcos y Lucas, habrían practicado otro calendario distinto al oficial del Segundo Templo (Humphhreys 2011, 49).

Ante este panorama, asumiendo la existencia de Jesús de Nazaret el problema general estará delimitado en este trabajo a investigar y determinar su probable calendario histórico, si es que lo hubo. Más no la fecha de su nacimiento.

\section{Planteamiento del primer problema previo.}

Pero a fin de abordar el problema general, se deben resolver otras interrogantes previas. En primer lugar, está el problema en determinar si se puede utilizar por sí solo el calendario judío moderno, como herramienta de investigación acerca de fechas de fiestas durante la vigencia del Segundo Templo.

Debe recordarse que el sabio Hillel II, decidió convocar al Sanedrín, previo a su abolición, e instituyó a mediados del siglo IV el actual calendario Judío. Para esa época fue un adelanto útil como una conjetura de punta para estimar fechas. Su originalidad fue optar por generar un calendario lunar alternativo al egipcio, al griego y demás conocidos para su tiempo. Diseñó un sistema que, dentro de ciclos de cada 19 años, mantiene a los años $1^{\circ}, 2^{\circ}, 4^{\circ}, 5^{\circ}, 7^{\circ}, 9^{\circ}, 10^{\circ}, 12^{\circ}, 13^{\circ}, 15^{\circ}, 16^{\circ}$ y $18^{\circ}$ de doce meses, y los numerales $3^{\circ}, 6^{\circ}, 8^{\circ}, 11^{\circ}, 14^{\circ}, 17^{\circ}$ y $19^{\circ}$, de trece meses lunares, en ciclos perpetuos.

No obstante, ese sistema presenta discordancias con prácticas que se realizaban en el Segundo Templo de Jerusalén de las lunas nuevas. Ello explica las divergencias dentro del judaísmo (Ajdler 2015, 133 190), y el propio resurgimiento a posterior de otros calendarios judíos como el "caraíta".

Por otro lado, con la destrucción del Templo en el año 70, se produjo una discontinuidad en la intercalación de un décimo tercer mes en adelante, afectando a posteriores resultados hasta el tiempo en que aparecerá Hillel II con su calendario: 


\section{Journal of the Academy $|85|$}

...Debido a la omisión de la intercalación durante un periodo prolongado de tiempo, en el año 137 se intercalaron tres años consecutivos de trece meses. También hay que señalar que era costumbre prohibir el mes decimotercero en los años sabáticos o en el año siguiente. La irregularidad en la intercalación originaba que dos años pudieran ser declarados embolísmicos y se llegó a establecer una regla para evitar la intercalación en tres años seguidos... (Segura 2017, 10).

Antes de continuar, en adelante los nombres de los meses del calendario judío moderno, numerados del $1^{\circ}$ al $12^{\circ}$, para los efectos de este trabajo serán: nisán, iyar, siván, tammuz, ab, elul, tishri, heshván, kislev, tevet, shevat, adar. Y para años con mes $13^{\circ}$, será el "segundo adar" ("adar shení").

Las fechas que se indicarán serán bajo la cuenta del calendario gregoriano, salvo expresa mención en contrario.

Se asumirá que los diversos textos de los cuatro evangelios son relatos sinceros de testigos oculares, o de oídas. Se trabajará sobre la idea de considerar que cuando mencionaban a cualquier "parte" de tiempo de una cierta unidad de día, mes o año, ellos contabilizaban esa misma parte o fracción, sea de "día", "mes" o "año", de forma completa. Y además que, en cada cómputo de tiempo, se iniciaba la cuenta desde esa misma parte o fracción como un día, un mes, o un año, entero, ya cumplido. Por otro lado, que los días "sábado" eran para ellos los días de descanso o feriados religiosos. Finalmente, se asumirá un ministerio público de la vida pública de Jesús, de alrededor de 3 años y medios.

A continuación, el planteamiento para los siguientes efectos será que el calendario judío de Hillel II es inseguro para investigar pascuas y otras fiestas antiguas. Para confirmar o descartarlo se hizo un experimento. Hace algunos años la revista de ciencias "Nature" publicó una investigación de Humphreys y Waddington (1983), dónde estos autores concluyen ciertas fechas de pascuas, con resultados similares a otras investigaciones (Fotheringham 1934). De acuerdo a eso, la prueba consistió en comparar fechas de meses desde mes de nisán al otro año y mes de nisán según el calendario judío con los cálculos de estos científicos por el periodo concreto investigado desde el 26 al 35, vinculado a fechas del calendario gregoriano. Los resultados fueron los siguientes:

\begin{tabular}{|l|c|c|c|c|}
\hline \multicolumn{5}{|c|}{ Tabla I: } \\
\hline $\begin{array}{l}\text { Fechas } \\
\text { de } \\
\text { Pascua }\end{array}$ & $\begin{array}{c}\text { Día } \\
\text { inicial } \\
\text { del año } \\
\text { (JDE) }\end{array}$ & $\begin{array}{c}\text { Día } \\
\text { final del } \\
\text { año } \\
\text { (JDE) }\end{array}$ & $\begin{array}{c}\text { M } \\
\text { Días } \\
\text { total }\end{array}$ & $\begin{array}{c}\text { e } \\
\text { s } \\
\text { e } \\
\text { s }\end{array}$ \\
\hline $\begin{array}{l}\text { Viernes } \\
20 / 3 / 26\end{array}$ & 1730622 & 1731004 & 382 & 13 \\
\hline $\begin{array}{l}\text { Miércoles } \\
7 / 4 / 27\end{array}$ & 1731005 & 1731359 & 354 & 12 \\
\hline $\begin{array}{l}\text { Lunes } \\
27 / 3 / 28\end{array}$ & 1731360 & 1731742 & 382 & 13 \\
\hline $\begin{array}{l}\text { Sábado } \\
14 / 4 / 29\end{array}$ & 1731743 & 1732096 & 353 & 12 \\
\hline $\begin{array}{l}\text { Miércoles } \\
3 / 4 / 30\end{array}$ & 1732097 & 1732451 & 354 & 12 \\
\hline $\begin{array}{l}\text { Lunes } \\
24 / 3 / 31\end{array}$ & 1732452 & 1732836 & 384 & 13 \\
\hline $\begin{array}{l}\text { Lunes } \\
12 / 4 / 32\end{array}$ & 1732837 & 1733190 & 353 & 12 \\
\hline $\begin{array}{l}\text { Viernes } \\
1 / 4 / 33\end{array}$ & 1733191 & 1733543 & 352 & 12 \\
\hline $\begin{array}{l}\text { Lunes } \\
20 / 3 / 34\end{array}$ & 1733544 & 1733928 & 384 & 13 \\
\hline $\begin{array}{l}\text { Lunes } \\
9 / 4 / 35\end{array}$ & 1733929 & 1734282 & 353 & 12 \\
\hline
\end{tabular}

\begin{tabular}{|l|l|l|l|l|}
\hline \multicolumn{5}{|c|}{ Tabla II: } \\
\hline $\begin{array}{l}\text { Fatos según Humps } \\
\text { de } \\
\text { Pascua }\end{array}$ & $\begin{array}{c}\text { Día No } \\
\text { inicial } \\
\text { del año } \\
\text { (JDE) }\end{array}$ & $\begin{array}{c}\text { Día No } \\
\text { final del } \\
\text { año } \\
\text { (JDE) }\end{array}$ & $\begin{array}{c}\text { Día } \\
\text { total }\end{array}$ & $\begin{array}{c}\text { e } \\
\text { s } \\
\text { e } \\
\text { s }\end{array}$ \\
\hline $\begin{array}{l}\text { Domingo } \\
\text { 19/4/26 }\end{array}$ & 1730652 & 1731005 & 353 & 12 \\
\hline $\begin{array}{l}\text { Jueves } \\
8 / 4 / 27\end{array}$ & 1731006 & 1731360 & 354 & 12 \\
\hline $\begin{array}{l}\text { Martes } \\
28 / 3 / 28\end{array}$ & 1731361 & 1731744 & 383 & 13 \\
\hline $\begin{array}{l}\text { Lunes } \\
16 / 4 / 29\end{array}$ & 1731745 & 1732098 & 353 & 12 \\
\hline $\begin{array}{l}\text { Viernes } \\
5 / 4 / 30\end{array}$ & 1732099 & 1732452 & 353 & 12 \\
\hline $\begin{array}{l}\text { Martes } \\
25 / 3 / 31\end{array}$ & 1732453 & 1732835 & 382 & 13 \\
\hline $\begin{array}{l}\text { Domingo } \\
11 / 4 / 32\end{array}$ & 1732836 & 1733190 & 354 & 12 \\
\hline $\begin{array}{l}\text { Viernes } \\
1 / 4 / 33\end{array}$ & 1733191 & 1733545 & 354 & 12 \\
\hline $\begin{array}{l}\text { Miércoles } \\
22 / 3 / 34\end{array}$ & 1733546 & 1733929 & 383 & 13 \\
\hline $\begin{array}{l}\text { Martes } \\
12 / 4 / 35\end{array}$ & 1733930 & 1734283 & 353 & 12 \\
\hline
\end{tabular}




\section{Journal of the Academy $|86|$}

Como se aprecia, salvo una luna nueva y la pascua del año 33, los demás resultados son dispares en cantidades totales, fechas y años, y por tanto es inseguro utilizar el calendario judío.

\section{Planteamiento y resolución de algunos problemas.}

Pero si el moderno calendario judío no sirve como herramienta para investigar fechas antiguas, la pregunta obligada es ¿cómo determinar otra herramienta de mayor seguridad para obtener fechas según el antiguo calendario ritual?

Y para responder tal incógnita, hay que replantearse otras nuevas preguntas anteriores, a saber, ¿cómo saber las fechas de las antiguas lunas nuevas?, y ¿cómo intercalar años de doce o de trece meses? Debido a estos problemas, se elaboró el siguiente plan de trabajo en la investigación:

a) Respeto del problema de las lunas nuevas:

El primer paso para avanzar se inició levantando información y recolección de datos astronómicos. Se registraron en planillas de cálculo Excel fechas por cada día, mes, y año del calendario gregoriano en el periodo comprendido entre el 24 de noviembre del -4713 al 16 de septiembre de 2240, por su número de orden científico de día juliano (JDE). Luego se superpusieron en paralelo a las fechas del antiguo calendario juliano. Se reordenaron 2.539.462 días en semanas de lunes a domingo según el uso moderno occidental en calendario gregoriano.

De los trabajos del meteorólogo y matemático Jean Meeus, el científico Fred Espenak ha elaborado y publicado importantes resultados astronómicos acerca del movimiento lunar proporcionados a través del Six Milenium Moon Events de la NASA. Sus resultados de datos en fechas, horas y fases lunares, junto a otras fuentes se emplearon para el desarrollo de la presente investigación.

El paso siguiente fue importar los resultados de aquellos datos, para luego concatenarlos a su respectiva fecha científica. Tras ello, se reajustó el tiempo universal del meridiano de Greenwich más (+) 2:00 horas a las cifras dadas del observatorio naval de EE.UU. debido a la diferencia entre el tiempo del lugar de la zona horaria de Tierra Santa con respecto a la hora Greenwich.

Por cada día anual se tomaron promedios de horarios diarios de atardecer a la zona de Tierra Santa, y se proyectaron a lo largo de los años y siglos. Luego se procedió a la integración de la información en un sistema virtual de trabajo.

El siguiente paso fue idear alguna manera de cómo dividir meses lunares e identificar fechas de lunas nuevas. En general se puede indicar que es dificultoso resolver este problema. Debido a costumbres prácticas dispares de avistamiento de las lunas nuevas, se llegan a fechas irregulares cada mes. Las dificultades se deben a diferencias geográficas, atmosféricas y especialmente a divisiones interpretativas acerca de textos sagrados entre los grupos.

Para abordar este problema, el punto de partida inicial fue observar que bajo la cultura hebrea, a groso modo, se pueden identificar a un cierto grupo, principalmente fariseos y la corriente rabínica que utilizan por regla general el criterio de fijar luna nueva el día siguiente al de la conjunción astronómica (salvo algunas ocasiones que lo hacen hasta el día subsiguiente). Esta posición, da como resultado que su luna llena caiga en $15^{\circ}$ del mes lunar por regla general.

En cambio, otros grupos, como por ejemplos saduceos y actualmente más proclives a judíos caraítas, utilizan por regla general el criterio del día subsiguiente al de la conjunción astronómica (salvo excepciones que lo hacen al día siguiente). Y bajo otra postura, a diferencia de la anterior, se da como resultado que la luna llena recaiga en día $14^{\circ}$ de cada mes por regla general.

La razón de este último criterio es, principalmente a que busca un primer filo lunar a simple vista de los ojos. Dos testigos a lo menos que hayan avistado de este modo la luna, debían concurrir ante el Tribunal 


\section{Journal of the Academy | $87 \mid$}

del antiguo Templo. Allí, se conformaría una audiencia. Una vez juramentados, se efectuarían previamente preguntas para establecer razones para aceptar el testimonio de un testigo. En caso de pasar este escollo, a continuación, someter al declarante a diversas interrogaciones del lugar del avistamiento, hora, condiciones, circunstancias, y en general, dar fiabilidad a los dichos. Acto seguido, lo mismo con los otros testigos. En caso de ser aceptados los testimonios, se declararía el día de la luna nueva.

El expediente de esta clase de ejercicios, conduciría a una proclama más acorde al día subsiguiente al de una conjunción, salvo excepciones.

Lo propio si al día $30^{\circ}$ del mes lunar, nadie alcanzaba a llegar para declarar en la Corte, o el número de testigos era insuficiente, o no eran considerados aptos para testimoniar, se pasaba a declarar el día siguiente, como luna nueva.

Ante este escenario, para el desarrollo de este trabajo, se privilegió la segunda postura, debido a su mayor generalidad práctica y vinculación con grupos saduceos, como Anás y Caifás, que lideraban en el Segundo Templo en tiempos bíblicos. Y de acuerdo a esto, la hipótesis que se formuló, fue que, la hora de la luna llena antes de la puesta de sol determina el día $14^{\circ}$ lunar. A partir del día $14^{\circ}$ para una fecha dada, se resta el numero 13 (días), y resulta el $1^{\circ}$ de cada mes. Si, en cambio, la hora de la luna llena es después de la puesta de sol determina ese día en lugar $13^{\circ}$ lunar. A partir de allí, se resta el numero 12 (días), para identificar el día $1^{\circ}$ del mes. Debe recordarse que el "día", en el calendario hebreo, inicia en la puesta de sol del día anterior, hasta la siguiente puesta de sol. Bajo esas ideas, la luna llena, permite indirectamente reconocer lunas nuevas.

Con eso, se realizaron diversas pruebas, con resultados positivos, comparando con prácticas actuales de judíos caraítas. De este modo, se despejaron las incógnitas de lunas nuevas en la historia. Y cada mes dividido en grupos de 29 , o de 30 días.

\section{b) Respecto del problema en la intercalación de meses trece (II Adar):}

El siguiente paso fue idear alguna manera de intercalar meses lunares. Para estos efectos, se observó el siguiente hecho: poco antes de la crucifixión, en Jn. 11:6 hay una mención muy inusual, de un Jesús que opta por descansar durante "dos" días consecutivos, desde que es informado de un urgente llamado de auxilio. Y, al siguiente día el autor de la carta recuerda la mención de Jesús afirmando que, dado que estaba en un día "de doce horas", era de aprovechar a fortiori la más luz día para hacer el bien $(J n$ 11,9).

Para aquella época, era sabido que el equinoccio de primavera es uno de los días en el año que, positivamente, tiene 12 horas de duración la luz en el día, y la noche.

Sí, a continuación, lo que estaba diciéndose era un día equinoccio de primavera, con más luz a los días anteriores al cabo de concluir el invierno, la hipótesis propuesta fue que debería existir alguna fecha límite máxima de dicho equinoccio con algún día lunar dado. Con esa idea obtenida de los textos antiguos, el paso siguiente fue recolectar y registrar las fechas de los días de equinoccios de primavera por cada año que se obtienen de los resultados en estudios especializados. En tal sentido los investigadores han usado las fórmulas de Jean Meeus para calcular los inicios de las fechas de equinoccios y solsticios en las estaciones desde el año -1000 al 2999.

A continuación, durante el curso del presente estudio, se recalcularon las fechas matemáticas que restaban de equinoccios y solsticios por el periodo desde el año -4713 al -1001 . Con todo ello, se registraron y compararon estas fechas de cada equinoccio en un periodo de 6.953 años. Se probaron equinoccios en diferentes días de fechas lunares.

Distintas pruebas virtuales dieron resultados negativos. Los años de doce o de trece meses, perdían sincronía. Como efecto dominós, las fechas de pascuas y demás efemérides, presentaban desfases e inconsistencias. 


\section{Journal of the Academy $|88|$}

Pero después de diversos experimentos, hubo una manera matemática que resulto para dividir años de 12 , o de 13 meses lunares. Una vez obtenida la fórmula, se procedió a probarla dentro de un rango mayor de años. Primero en 10, 30, 50 años. La fórmula es la siguiente:

Si un día de equinoccio de primavera coincide dentro de cualquier día de un mes de "adar" y aún hasta el día $3^{\circ}$ del siguiente mes que siga, este último mes pasará a ser "nisán" y no habrá necesidad de intercalación debido que a será un año normal, de doce meses; pero si esa fecha de equinoccio pasa del día $3^{\circ}$ y combina con el $4^{\circ}$ día lunar o recae en cualquier otro posterior en adelante este nuevo mes pasará a ser denominado segundo mes adar, habrá que hacer, necesariamente, una intercalación de un mes trece, y el año será llamado embolismal.

Finalmente, se experimentó en un universo de 2.539.462 días totales las reglas indicadas bajo esquema de programación condicional. Y a partir de la experimentación, se obtuvo una manera alternativa a la teoría tradicional del ciclo métonico readaptado de Hillel II, con resultados positivos, para intercalar.

\begin{tabular}{|c|c|c|c|}
\hline \multicolumn{4}{|c|}{$\begin{array}{c}\text { Tabla III } \\
\text { Datos desde el calendario Judío }\end{array}$} \\
\hline $\begin{array}{c}\mathbf{N}^{\circ} \\
\text { Año } \\
\text { de } \\
\text { Ciclo }\end{array}$ & $\begin{array}{l}\text { Año } \\
\text { Judío }\end{array}$ & $\begin{array}{l}\text { Fechas de } \\
\text { Pascuas }\end{array}$ & $\begin{array}{l}\mathbf{M} \\
\mathbf{E} \\
\mathbf{S} \\
\mathbf{E} \\
\mathbf{S}\end{array}$ \\
\hline $1^{\circ}$ & 3782 & $\begin{array}{l}\text { Sábado } \\
02 / 04 / 22\end{array}$ & 12 \\
\hline $2^{\circ}$ & 3783 & $\begin{array}{l}\text { Miércoles } \\
22 / 03 / 23\end{array}$ & 12 \\
\hline $3^{\circ}$ & 3784 & $\begin{array}{l}\text { Miércoles } \\
10 / 04 / 24\end{array}$ & 13 \\
\hline $4^{\circ}$ & 3785 & $\begin{array}{l}\text { Lunes } \\
31 / 03 / 25 \\
\end{array}$ & 12 \\
\hline $5^{\circ}$ & 3786 & $\begin{array}{l}\text { Viernes } \\
20 / 03 / 26\end{array}$ & 12 \\
\hline $6^{\circ}$ & 3787 & $\begin{array}{l}\text { Miércoles } \\
07 / 04 / 27 \\
\end{array}$ & 13 \\
\hline $7^{\circ}$ & 3788 & $\begin{array}{l}\text { Lunes } \\
27 / 03 / 28\end{array}$ & 12 \\
\hline $8^{\circ}$ & 3789 & $\begin{array}{l}\text { Sábado } \\
14 / 04 / 29\end{array}$ & 13 \\
\hline $9^{\circ}$ & 3790 & $\begin{array}{l}\text { Miércoles } \\
03 / 04 / 30\end{array}$ & 12 \\
\hline $10^{\circ}$ & 3791 & $\begin{array}{l}\text { Lunes } \\
24 / 03 / 31 \\
\end{array}$ & 12 \\
\hline $11^{\circ}$ & 3792 & $\begin{array}{l}\text { Lunes } \\
12 / 04 / 32\end{array}$ & 13 \\
\hline $12^{\circ}$ & 3793 & $\begin{array}{l}\text { Viernes } \\
01 / 04 / 33\end{array}$ & 12 \\
\hline $13^{\circ}$ & 3794 & $\begin{array}{l}\text { Lunes } \\
20 / 03 / 34\end{array}$ & 12 \\
\hline $14^{\circ}$ & 3795 & $\begin{array}{l}\text { Lunes } \\
09 / 04 / 35\end{array}$ & 13 \\
\hline $15^{\circ}$ & 3796 & $\begin{array}{l}\text { Viernes } \\
28 / 03 / 36\end{array}$ & 12 \\
\hline $16^{\circ}$ & 3797 & $\begin{array}{l}\text { Jueves } \\
18 / 03 / 37\end{array}$ & 12 \\
\hline
\end{tabular}

\begin{tabular}{|l|c|c|c|c|}
\hline \multicolumn{5}{|c|}{ Tabla IV } \\
\hline $\begin{array}{c}\text { Fechas de } \\
\text { Pascuas }\end{array}$ & $\begin{array}{c}\text { E } \\
\text { S } \\
\text { E } \\
\text { S }\end{array}$ & $\begin{array}{c}\text { Día N } \\
\text { inicial } \\
\text { del año } \\
\text { (JDE) }\end{array}$ & $\begin{array}{c}\text { Día N }^{\circ} \\
\text { final } \\
\text { del año } \\
\text { (JDE) }\end{array}$ & $\begin{array}{c}\text { Días } \\
\text { del } \\
\text { año }\end{array}$ \\
\hline $\begin{array}{l}\text { Domingo } \\
\text { 03/04/22 }\end{array}$ & 12 & 1728997 & 1729350 & 353 \\
\hline $\begin{array}{l}\text { Sábado } \\
22 / 4 / 23\end{array}$ & 13 & 1729351 & 1729734 & 383 \\
\hline $\begin{array}{l}\text { Jueves } \\
11 / 4 / 24\end{array}$ & 12 & 1729735 & 1730089 & 354 \\
\hline $\begin{array}{l}\text { Lunes } \\
31 / 3 / 25\end{array}$ & 12 & 1730090 & 1730444 & 354 \\
\hline $\begin{array}{l}\text { Sábado } \\
18 / 4 / 26\end{array}$ & 13 & 1730445 & 1730828 & 383 \\
\hline $\begin{array}{l}\text { Jueves } \\
08 / 4 / 27\end{array}$ & 12 & 1730829 & 1731182 & 353 \\
\hline $\begin{array}{l}\text { Martes } \\
25 / 4 / 28\end{array}$ & 13 & 1731183 & 1731566 & 383 \\
\hline $\begin{array}{l}\text { Domingo } \\
15 / 04 / 29\end{array}$ & 12 & 1731567 & 1731920 & 353 \\
\hline $\begin{array}{l}\text { Viernes } \\
05 / 4 / 30\end{array}$ & 12 & 1731921 & 1732274 & 353 \\
\hline $\begin{array}{l}\text { Jueves } \\
24 / 4 / 31\end{array}$ & 13 & 1732275 & 1732658 & 383 \\
\hline $\begin{array}{l}\text { Lunes } \\
12 / 4 / 32\end{array}$ & 12 & 1732659 & 1733013 & 354 \\
\hline $\begin{array}{l}\text { Viernes } \\
01 / 4 / 33\end{array}$ & 12 & 1733014 & 1733367 & 353 \\
\hline $\begin{array}{l}\text { Jueves } \\
20 / 4 / 34\end{array}$ & 13 & 1733368 & 1733751 & 383 \\
\hline $\begin{array}{l}\text { Lunes } \\
09 / 4 / 35\end{array}$ & 12 & 1733752 & 1734106 & 354 \\
\hline $\begin{array}{l}\text { Domingo } \\
27 / 04 / 36\end{array}$ & 13 & 1734107 & 1734490 & 383 \\
\hline $\begin{array}{l}\text { Jueves } \\
16 / 4 / 37\end{array}$ & 12 & 1734491 & 1734844 & 353 \\
\hline
\end{tabular}


Journal of the Academy $|89|$

\begin{tabular}{|c|c|l|c|}
$17^{\circ}$ & 3798 & $\begin{array}{l}\text { Lunes } \\
05 / 04 / 38\end{array}$ & 13 \\
\hline $18^{\circ}$ & 3799 & $\begin{array}{l}\text { Viernes } \\
25 / 03 / 39\end{array}$ & 12 \\
\hline $19^{\circ}$ & 3800 & $\begin{array}{l}\text { Viernes } \\
13 / 04 / 40\end{array}$ & 13 \\
\hline
\end{tabular}

\begin{tabular}{|l|c|c|c|c|}
$\begin{array}{l}\text { Martes } \\
06 / 4 / 38\end{array}$ & 12 & 1734845 & 1735198 & 353 \\
\hline $\begin{array}{l}\text { Lunes } \\
25 / 4 / 39\end{array}$ & 13 & 1735199 & 1735582 & 383 \\
\hline $\begin{array}{l}\text { Sábado } \\
14 / 4 / 40\end{array}$ & 12 & 1735583 & 1735936 & 353 \\
\hline
\end{tabular}

De este experimento, los resultados se aprecian en diferencias entre años de 12 y 13 meses, como las lunas nuevas, coincidiendo pocas pascuas. Por tanto, se concluye que no es seguro el calendario judío para estudiar los evangelios.

\section{Fecha de pascua de crucifixión del año 33 sometida a pruebas}

Pero para entender mejor estos resultados y sus implicancias, nuestro modelo debería servir para determinar algunos sucesos, como la fecha de pascua de crucifixión. El cuestionamiento es relevante debido a que descubrir la fecha, permitiría además saber a ciencia cierta la hora y la fase lunar en qué tuvo lugar.

Y sin asumir ni descartar ninguna fecha entre los años 26 al 36, en principio simplemente para partir con análisis, el paso siguiente fue someter a examen la posición que propugna la crucifixión en el día $1^{\circ}$ de abril del 33. Humphreys y Waddington propugnan que entre el 26 al 36 sólo en esa ocasión la pascua judía fue "viernes" y habría sido el día al que aludiría Pedro en su mensaje en pentecostés al citar un texto-prueba de una la luna roja.

Estiman que ese día hubo un eclipse lunar que inicio a las 15:50 hrs., alcanzando una máxima del 60\% alrededor de las 17:15 horas. Humphreys y Waddington llegan a igual conclusión que antes sostuvieron Newton, Fotheringham, y Bralley Schaefer.

Sin embargo, hay dudas que sea la crucifixión debido a que se discute si realmente pudo ser visible el eclipse desde Jerusalén. El argumento del texto prueba de un supuesto eclipse lunar puede servir, pero es insuficiente debido a que presupone que en la mente de Pedro estuviese la intención de dar cuenta de una efectiva luna roja en la crucifixión. Y hay investigadores que estiman que fue en día "miércoles".

No obstante, se puede tener presente que hay consenso en los cálculos expertos que el $1^{\circ}$ de abril del año 33 tuvo lugar la "luna llena" a las 14:52 horas UTC.

Con la finalidad de confirmar o descartar esa fecha, primero se realizó una cronología de los sucesos de Jesús, y a continuación se compararon diversas fechas de fiestas. En este sentido si se menciona la primera pascua en Jn. 2:13, y esa hubiere sido en el año 30, en este caso en viernes 5 de Abril; para el siguiente año, es consistente Jn. 5:9 que menciona que hubo una fiesta judía en "sábado", por el día 24 de mayo del 31 ("pascua segunda").

Y para el tercer año, si al día siguiente del último día de la fiesta de tabernáculos, hubiere sido el día "sábado" semanal (Jn. 8:2-9:14), para el 16 de octubre del 32, resultaría fechable el viernes 15 de octubre de ese año como día de fiesta aludido en Jn. 7:37. Finalmente, al cuarto año la pascua de crucifixión en el 1 de abril, antes del día "sábado" de reposo por el 2 de abril del 33.

De los experimentos entre los años 26 al 36, la conclusión de Humphreys y Waddington resulto una de las mejores, sin ser concluyente aún, a juicio nuestro.

\section{Del Calendario de Jesús histórico}

Para dar respuestas definitivas, y volver al planteamiento del problema central, el paso siguiente fue iniciar por reevaluar patrones de algunas ancestrales prácticas religiosas y culturales. Lo primero que se pudo observar es que, bajo la cosmovisión del pensamiento judeo cristiano, e incluso musulmán, está la práctica constante de mantener un día fijo, de reposo semanal, cada siete días un feriado de descanso 


\section{Journal of the Academy $|90|$}

semanal. Bajo ese paradigma se esconde el esquema de una suma repetitiva de cada 7 días descansar un séptimo día: +7 . Por tanto, en cualquiera de esos escenarios, lo que se observa es una simple sumatoria de "parar", descansar o detenerse "un día", cada séptimo.

La segunda observación: los evangelios describen en cambio a una tercera persona (Jesús de Nazaret) que en forma muy distinta, reposa o descansa durante dos o hasta el tercer día consecutivo.

En tercer lugar, se pudo también observar que cada fin de mes lunar desde que la luna se oculta, hasta que reaparece en el cielo a simple vista hay un rango de 2, o de 3 días de oscilación, que demora en aparecer su primer filo lunar, dependiendo, si el mes lunar es de 29 días, o de 30 días.

Producto de estas tres observaciones, surgió la siguiente hipótesis: Jesús llevaba otro calendario. Guardaba las lunas nuevas de cada mes, y según esos días, descansaba los siguientes 4 séptimos días semanales, en forma lunar. $\mathrm{O}$, lo que es equivalente a decir que él vivió guardando una secuencia lunar precisa por los días números $1^{\circ}, 8^{\circ}, 15^{\circ}, 22^{\circ}$ y $29^{\circ}$, todos los meses.

Debido a eso, cuando un mes dado resulta ser de 29 días, el séptimo día en la cuarta semana lunar (o, dicho de otro modo, ese último día $29^{\circ}$ del mes), era para él un día festivo, y permanecía en descanso. Pero era un primer día seguido de un segundo por el primer día del siguiente mes de la luna nueva.

En cambio, si el mes era de 30 días, el séptimo día en la cuarta semana lunar (o, dicho de otro modo, ese último día $29^{\circ}$ del mes), seguía para él siendo un día de descanso. Al cabo de ello, le seguía el día $30^{\circ}$. Y luego, al tercer día, se suma la luna nueva por el primer día del siguiente mes. Debido a esto, se lo describe en otras ocasiones distintas hasta "el tercer día" en algún lugar.

Para la comprobación de la hipótesis, se realizaron los siguientes nuevos pasos. Se revisó el periodo de los años 26 al 36, por cada fecha y día semanal, bajo las fórmulas antes propuestas. Luego se partió desde la fecha del viernes 1 de abril del 33, en una reconstrucción hacia atrás. Si se retrocede desde este día en busca de un equinoccio de primavera inmediatamente anterior, los datos muestran que tuvo lugar el domingo 20 de marzo del 33 a eso de las 16:01 horas, en nisán 2. Ese sería el día de 12:00 horas aludido en Jn. 11:9.

Si esta interpretación es correcta, habría que observar que aquellos "dos" días anteriores en descansos, que aparecen mencionados en Jn. 11,6, habrían sido los días viernes 18 y sábado 19 de marzo del 33, donde la cantidad de 29 días del mes y las fases lunares de la hipótesis fueron consistentes con los datos. Por otro lado, si de nuevo de parte desde la pascua del año 33 hacia atrás, la primera pascua mencionada en Jn. 2:13 habría sido en el viernes 5 de abril. A continuación, en iyar, la luna llena fue el sábado 4 de mayo a las 5:01 hrs.; en siván, cayó el domingo 2 de junio a las 12:10 hrs.; en tamuz, fue el lunes 01 de julio a las 20:01 hrs.; en el mes de av, el miércoles 31 de julio, a las 5:27 hrs.; en elul el jueves 29 de agosto a las 17:22 hrs.; y, en tisrhi, la luna llena fue el sábado 28 de septiembre a las 08:28 hrs., todos del año 30. En Jn. 4:35 y siguientes, se describe a Jesús que afirma en algún momento luego de la conversación con una samaritana que ese día era una fecha en que faltarían aún cuatro meses para la ciega, (que caería alrededor del mes de tisrhi). Por tanto, se observa que Jesús hablaría mientras eran los días del final del mes de tamuz.

Eso coincidió con los datos científicos, debido a que de nuevo el mes fue de 29 días. Por lógica consecuencia, descansaría luego dos días seguidos, como sigue mencionando el cuarto evangelio. En otras palabras, se fecharía por el martes 16 de julio del 30 se habría producido aquél encuentro.

Antes de la tercera fiesta de pascua mencionada en Jn. 6:4, que podríamos fechar por el lunes 12 de abril del 32 (nisán, 14, 3792 calendario judío), el testimonio escrito vuelve a referir "dos días" especiales. Y de nuevo, el mes lunar que precedió, fue de 29 días. Debido a eso, siguiendo la misma lógica anterior, estos días fueron los lunes 29 y martes 30 de marzo del 32.

En cambio, distinta es la situación cuando finalizaba en meses de 30 días lunares. Aquí los experimentos de comparar fechas, coinciden por el contrario con descansos hasta el "tercer día", como el caso en 


\section{Journal of the Academy $|91|$}

relación a una boda en Caná de Galilea (según Jn. 2:1). También, poco después de la pascua mencionada en Jn. 6:4, otros dos testigos declararán que Jesús es vuelto a permanecer en descanso hasta un "tercer día" (día 1 iyar) donde será visto (Marcos 8:2) en algún lugar de una montaña. Esto otra vez reflejaría el patrón debido a que ese mes de nisán fue de 30 días lunares. La luna nueva del jueves 29 de abril del 32 como ese "tercer día" que menciono Mateo (Mt 15:32).

Se debe tener presente que no se pretende un tratamiento de todos los casos con carácter exhaustivos. Hay otros. Simplemente es abrir una discusión y generar un nuevo camino de investigación.

Otro experimento: se compararon lunas nuevas en fiestas de Januka entre el 26 al 36 . Esto debido a que en Jn. 10:22 se mencionó a Jesús en el Templo un día de la fiesta de invierno. El resultado fue sintomático: otra vez coincidió esa fiesta con un día de luna nueva en 1 de Tevet. En este caso, el lunes 20 de diciembre del año 32, en forma consistente, con la secuencia cronológica de fechas y años.

Para revalidar la información obtenida, repetimos pruebas una y otra vez, para confirmar o descartar la fecha de crucifixión. El siguiente cuadro comparativo, resume otras fechas de pascuas de crucifixión, para evaluar cuáles, cumplían con más (+) o con menos (-) de los supuestos, según los textos: 
Journal of the Academy $|92|$

\begin{tabular}{|c|c|c|c|c|c|c|c|c|c|}
\hline \multicolumn{10}{|c|}{ Datos de la vida pública de Jesús Histórico } \\
\hline $\begin{array}{c}\mathbf{1}^{\circ} \\
\mathbf{A n ̃ o}\end{array}$ & $\begin{array}{c}\mathbf{2}^{\circ} \\
\text { Año }\end{array}$ & \multicolumn{7}{|c|}{$\begin{array}{c}\mathbf{3}^{\circ} \\
\mathbf{A n ̃ o}\end{array}$} & $\begin{array}{c}4^{\circ} \text { Año } \\
\text { ¿Crucifixión? }\end{array}$ \\
\hline $\begin{array}{l}1^{\text {a }} \text { Pascua Jn. } \\
2: 13\end{array}$ & $\begin{array}{l}2^{\text {a }} \text { Pascua } \\
\text { + fiesta en } \\
\text { sábado Jn. 5: } 9\end{array}$ & $\begin{array}{l}3^{\text {a }} \text { Pascua } \\
\text { de Jesús Jn. 6:4 }\end{array}$ & $\begin{array}{l}\text { Un último } \\
\text { día de Sucot en } \\
\text { viernes } \\
\text { Jn } 7: 37\end{array}$ & $\begin{array}{l}\text { Al otro día: } \\
\text { Sábado } \\
\text { Jn 8:2-9:14 }\end{array}$ & $\begin{array}{l}\text { Un día de fiesta } \\
\text { Janucá en luna } \\
\text { nueva } \\
\text { Jn 10:1 }\end{array}$ & $\begin{array}{l}\text { Un } 1^{\circ} \text { día, } 29 \\
\text { Adar Jn 11:6 }\end{array}$ & $\begin{array}{l}\text { Al } 2^{\circ} \text { día, } \\
\text { luna nueva, } 1^{\circ} \\
\text { nisán Jn. 11:6 }\end{array}$ & $\begin{array}{l}\mathrm{Al} 3^{\circ}: \text { día de } 12 \\
\text { horas } \\
\text { equinoccio Jn. } \\
11: 9\end{array}$ & $\begin{array}{l}4^{\text {a }} \text { Pascua } \\
\text { pasión } \\
\text { ¿Jn 19:31? }\end{array}$ \\
\hline Jueves $11 / 4 / 24$ & $(-)$ & $\begin{array}{l}\text { Sábado } \\
18 / 04 / 26\end{array}$ & \begin{tabular}{|l|}
$(+)$ \\
Viernes \\
$23 / 10 / 26$ \\
\end{tabular} & $\begin{array}{l}(+) \\
\text { Sábado } \\
24 / 10 / 26 \\
\end{array}$ & $(-)$ & $(-)$ & $(-)$ & $(-)$ & $\begin{array}{l}\text { Jueves } \\
8 / 4 / 27\end{array}$ \\
\hline $\begin{array}{l}\text { Sábado } \\
18 / 4 / 26\end{array}$ & $\begin{array}{l}(+) \\
\text { Sábado 8/5/27 } \\
\text { Pascua II } \\
\text { Shení } \\
\text { DL 15, Iyar } \\
\end{array}$ & Martes 25/4/28 & $(-)$ & $(-)$ & $(-)$ & $(-)$ & $(-)$ & $(-)$ & $\begin{array}{l}\text { Domingo } \\
15 / 4 / 29\end{array}$ \\
\hline $\begin{array}{l}\text { Jueves } \\
8 / 4 / 27\end{array}$ & $\begin{array}{l}(+) \\
\text { Sábado } \\
21 / 10 / 28 \\
\text { Pascua II } \\
\text { Shení, } \\
\text { DL 15, Iyar } \\
\end{array}$ & $\begin{array}{l}\text { Domingo. } \\
15 / 4 / 29\end{array}$ & $\begin{array}{l}(+) \\
\text { Viernes } \\
19 / 10 / 29\end{array}$ & $\begin{array}{l}(+) \\
\text { Sábado } \\
\text { 20/10/29 }\end{array}$ & $\begin{array}{l}(+) \\
\text { 24/12/29 } \\
\text { Tevet Januká } 6 \\
\text { En luna nueva }\end{array}$ & $(-)$ & $(-)$ & $(-)$ & $\begin{array}{l}\text { Viernes } \\
5 / 4 / 30\end{array}$ \\
\hline $\begin{array}{l}\text { Martes } \\
25 / 4 / 28\end{array}$ & $(-)$ & Viernes $5 / 4 / 30$ & $\begin{array}{l}(-) \\
\text { Lunes } 7 / 10 / 30\end{array}$ & $\begin{array}{l}(-) \\
\text { Martes 8/10/30 }\end{array}$ & $\begin{array}{l}(+) \\
\text { 14/12/30 Tevet } \\
\text { Januká } 6 \text { en } \\
\text { luna nueva } \\
\end{array}$ & $(-)$ & $(-)$ & $(-)$ & Jueves $24 / 4 / 31$ \\
\hline Viernes $5 / 4 / 30$ & $\begin{array}{l}(+) \\
\text { Sábado } \\
24 / 5 / 31 \\
\text { Pascua II } \\
\text { Shení } \\
\text { DL 15, Iyar }\end{array}$ & Lunes $12 / 4 / 32$ & $\begin{array}{l}(+) \\
\text { Viernes } \\
15 / 10 / 32\end{array}$ & $\begin{array}{l}(+) \\
\text { Sábado } \\
\text { 16/10/32 }\end{array}$ & $\begin{array}{l}(+) \\
\text { 20/12/32 Tevet } \\
\text { Januká } 4 \text { en } \\
\text { luna nueva }\end{array}$ & $\begin{array}{l}(+) \\
\text { viernes 18/3/33 } \\
\text { DL } 29 \text { Adar } 29\end{array}$ & $\begin{array}{l}(+) \\
\text { sábado 19/3/33 } \\
\text { DL } 1 \text { Nisán }\end{array}$ & $\begin{array}{l}(+) \\
\text { domingo } \\
20 / 3 / 33 \\
\text { DL 2 } \\
\text { Nisán } \\
\text { equinoccio } \\
\text { Primavera }\end{array}$ & $\begin{array}{l}(+) \\
\text { Viernes } 01 / 4 / 33 \\
\& \\
\text { Eclipse } \\
\text { Lunar parcial }\end{array}$ \\
\hline
\end{tabular}




\section{Journal of the Academy $|93|$}

De acuerdo a esta última tabla, se puede observar que se cumplen los supuestos (+) que apuntan en favor de la pascua de crucifixión en el 33. Y a esta última fecha habría que agregar todas las demás descripciones de "dos" días en descanso con los samaritanos, faltando cuatro meses para la ciega, a los dos días antes de la tercera pascua o "al tercer día" de fines del mismo mes de nisán e inicio de iyar (debido al último día del mes, más el inició de las lunas nuevas) que fueron antes explicitadas y que se corresponden en paralelo con el movimiento lunar; lo que marca otras diferencias para privilegiar el año 33.

Se pudo observar que no hubo fiesta de pascua en "miércoles", entre el 26 al 36.

La fecha del viernes 1 de abril del 33 hay más de 15 supuestos que se cumplen, lo que obliga a confirmar que la conclusión defendida por los científicos antes nombrados es más consistente, con la metodología del calendario que ha sido propuesta, a diferencia de las demás fechas divergentes.

Finalmente, los resultados llevan a proponer un esquema ideal del calendario mensual concebido y observado por Jesús, que para la fecha de su crucifixión, habría quedado bajo la siguiente representación gráfica:

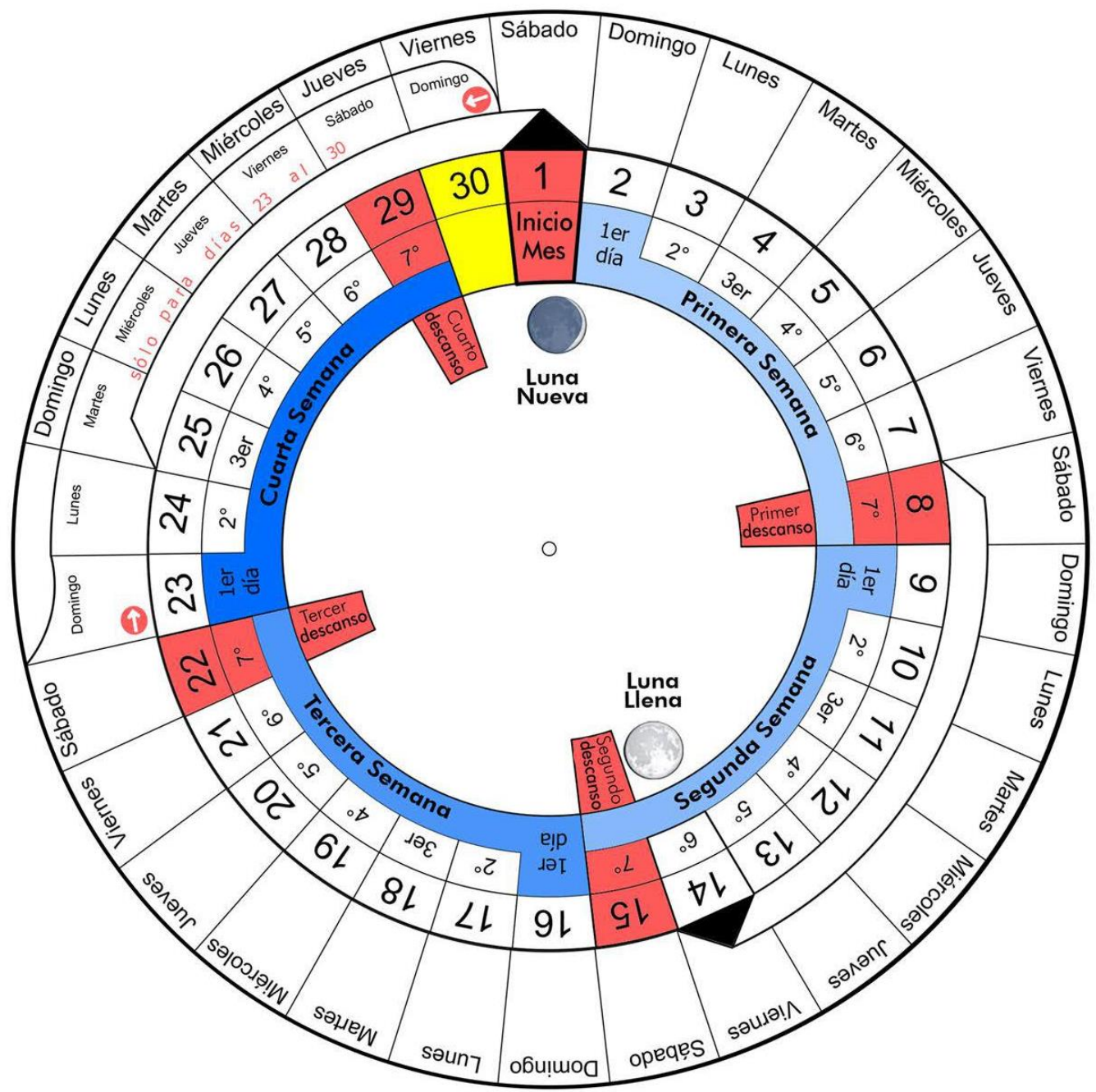




\section{Journal of the Academy $|94|$}

\section{CONCLUSIONES}

De acuerdo a lo investigado, se puede estimar que no es seguro utilizar el calendario Judío para analizar fechas antiguas. Aunque fue un aporte para su tiempo al readaptar el sistema griego al hebreo, el experimento de comparar con la investigación de Humphreys y Waddington muestra inconsistencias. Para superar sus deficiencias se puede concluir, por un lado, que ayuda el criterio de la luna llena como día $14^{\circ}$ y por el otro, un mejor método para saber qué años fueron de doce, o de trece meses, es aplicar la regla de la fecha límite del día de equinoccio de primavera que fue postulado.

Bajo esta metodología se puede concluir que las fiestas que resultan son consistentes con los datos que aporta el Nuevo Testamento para el periodo del año 29 al 33, y obliga a confirmar la crucifixión en el viernes 01 de abril del 33.

El autor del cuarto evangelio hablando a ciegas recordó días de lunas nuevas y descansos precisos de Jesús, que son compatibles con las modernas bases astronómicas. Juan fue un testigo ocular de primera mano de los hechos.

Sus registros detallan implícitamente un calendario diferente de Jesús que permite reconocerlo como persona histórica. Su propio modo práctico de vivir escondía una manera simple y útil para usar en otro nuevo método de datación histórica. Los esquemas de fechas y su manera de computar el tiempo se pueden implicar matemática y astronómicamente a lo largo de la historia. Incluso, retroproyectar en paralelo con cualquier otro calendario científico.

Los resultados finales de esta investigación, no alcanzan a contenerse en un texto debido a la inmensidad de datos que implicaría. Por lo anterior, se ideo su presentación en un sistema de lenguaje en PHP, $\mathrm{Html}$, y otros programas de diseño afines, para consulta de fechas bajo el sitio www.elcalendario.org

Las implicancias de esta investigación son insospechadas. Desde revolucionar a las ciencias bíblicas, o revivir otros calendarios antiguos para rehacer otras fechas históricas, ayudar en usos terapéuticos, o incluso hasta la investigación que relaciona delitos con fases lunares para mejorar políticas publicas contra la delincuencia, y otros variados campos de estudio.

\section{REFERENCIAS BIBLIOGRÁFICAS}

Ajdler, J. Jean (2015). A Short History of the Jewish Fixed Calendar: The Origin of the Molad. Hakira, the Flatbush Journal of Jewish Law and Throat, 133-190.

Alvarez, Cristhian. (2011). ¿23 de Septiembre o 22 de Octubre? Xápis, 3-19.

Bacchiocchi, Samuele. (1980). Reposo Divino para la Inquietud humana. Michigan.

Ben-Dov, Jonathan. (2008). Head of All Years: Astronomy and Calendars at Qumran in Their Ancient Context. Leiden, Boston: Brill.

(2012). Lunar Calendars at Qumran?: A Comparative and Ideological Study. En Living the Lunar Calendar, 173-190.

Bonavoglia, Paolo. (2000). Eclissi e Calendari. Recuperado el 30 de julio de 2019, de Calendario Perpetuo: http://astro.bonavoglia.eu/

Cantera, F.; Iglesias, M. (2009). Sagrada Biblia, versión crítica sobre los textos hebreo, arameo y griego. Madrid: Biblioteca de Autores Cristianos. 


\section{Journal of the Academy $\mid 95$ |}

Carabias, Ana María. (2012). Salamanca y la medida del tiempo. Salamanca: Universidad de Salamanca.

Castillo, Samuel. (2001). Equivalencia entre el Calendario Lunar y el Calendario Gregoriano. Theoría, 10, 33-37.

Espenak, Fred. (2012). astropixels. Recuperado el 30 de septiembre de 2016, de http://www.astropixels.com/

Espenak, Fred; Meeus, Jean. (Enero de 2009). Five Millennium Catalog of Solar Eclipses: -1999 to +3000 (2000 BCE to 3000 CE) - Revised. Recuperado el 4 de septiembre de 2019, de NASA ECLIPSE WEB SITE: https://eclipse.gsfc.nasa.gov/SEpubs/5MCSE.html

Fotheringham, John. (1934). The Evidence of Astronomy and Technical Chronology for the Date of the Crucifixion. Journal of Theological Studies, 146-162.

García, S. A. (1998). La datación histórica. Navarra: EUNSA, Ediciones Universidad de Navarra.

Humphhreys, Colin. (2011). The mystery of the last supper. Reconstructing the final Days of Jesus. Cambridge: University.

Humphreys, Colin; Waddington, W.G. (1983). Dating the Crucifixion. Nature, 743-46.

(1992). The jewish calendar, a lunar eclipse and the date of Christ's Crucifixion. Tyndale Bulletin 43.2, 331-351.

(2017). Solar eclipse of 1207 BC helps to date pharaohs. Recuperado el 14 de septiembre de 2019, de Astronomy \& Geophysics, V 58, No 5: https://academic.oup.com/astrogeo/article/58/5/5.39/4159289

Jaubert, Annie. (1965). The Date of the Last Supper. Nueva York: Alba House.

Kontakt, (s.f.). beda. Recuperado el 30 de noviembre de 2016, de https://www.beda.cz/ jirkaj/seasons/ Landman, Isaac. (1901-1906). The Universal Jewish Encyclopedia (Vols. 5, sec. Holidays). New York: Ktav Publishing House.

Lull, José. (2006). La Astronomía en el antiguo Egipto. Valencia: Universidad de Valencia.

Meeus, Jean. (1985). Astronomía Con il Computer. Milano: Hoepli.

Meier, John. (1998). Un judío Marginal. Nueva Visión del Jesús histórico. Tomo I. Navarra: Verbo Divino.

Nasa, C. (s.f.). Recuperado el 3 de julio de 2019, de https://eclipse.gsfc.nasa.gov/eclipse.html

Piñero, Antonio. (2006). Guía para entender el Nuevo Testamento. Madrid: Trotta.

(2009). Todos los Evangelios. Traducción integra de las lenguas originales de todos los textos evangélicos conocidos. Madrid: digital Titivillus.

Pratt, John. (1991). Newton's Date for the Crucifixion. Royal Astronomical Society, 32 Núm. 3, 301304.

Ratzinger, Joseph. (2011). Jesús de Nazaret II. Vaticana: Morgan Editores. 


\section{Journal of the Academy $|96|$}

Roitman, Adolfo. (2010). Biblia, exégesis y religión. Una Lectura crítico-histórica del judaísmo. Navarra: Verbo Divino.

(Diciembre de 2013). Juan Bautista, Jesús, Pablo y la Comunidad de Qumrán, en Cuadernos Judaicos. Revista Académica de la Universidad de Chile. Recuperado el 14 de septiembre de 2019, de https://cuadernosjudaicos.uchile.cl/

Sanders, E. P. (2000). La figura histórica de Jesús. Navarra: Verbo Divino.

(2004). Jesús y el Judaísmo. (J. Pérez, Trad.) Madrid: Trotta.

Saulnier, Stéphanier. (2012). Calendrical Variations in Second Temple Judaism. Leiden - Boston: Brill.

Segura, Wenceslao. (2017). Calendario Hebreo. España, WT Ediciones.

Schaefer, Bradley. (1990, March). Lunar visibility and the crucifixión. Royal Astronomical Society, 31(1), 53-67.

Schiffman, L. H.; VanderKam, J.C. (2000). Calendars and Mishmarot. En Encyclopedia of the Dead Sea Scrolls (Vol. I). Oxford University Press.

Velasco, J. Martín. (1978). Introducción a la Fenomenología de la Religión. Madrid: Ediciones Cristiandad. 